\title{
Extremadura: política regional y dependencia (1986-1999)
}

\section{Marcelo Sánchez-Oro Sánchez \\ Universidad de Extremadura msanoro@unex.es}

Con este trabajo hemos querido obtener una comprensión del entramado de recursos, instrumentos y políticas que han orientado, en un periodo de tiempo determinado, las acciones en favor de las regiones más atrasadas de Europa y cómo se implementan en Extremadura. Específicamente se ha tratado de analizar las políticas regionales aplicadas en la región y evaluar su impacto, para lo cual hemos recurrido a las series de datos cuantitativos disponibles. Otra pretensión de este trabajo fue realizar una valoración de la incidencia del modelo de desarrollo por el que optan las instituciones regionales. Este segundo objetivo obligó a realizar una valoración crítica del paradigma de desarrollo dominante, entendido como aquel que, frente a otras posibilidades, se impone en los procesos de toma de decisiones político-administrativas. Estos objetivos no podrían lograrse sin examinar la estructura social extremeña y determinar las bases en las que se asienta el actual sistema de dominación.

Palabras clave: Desarrollo Regional, Política Regional, Modelos de Desarrollo, Extremadura, Dependencia Política.
Our aim with this piece of work has been to achieve two main goals: in the first place, to obtain a thorough comprehension of the network of resources, instruments and policies that for a given period of time have guided the action taken in favor of the depressed regions, and more precisely Extremadura. In specific terms, an attempt has been made to analyse the regional policies applied in the region and to evaluate their impact, and for this we have resorted to an appraisal of the incidence of the development model that regional institutions choose. On the other hand, we wanted to make a critical evaluation of the dominant development model, understanding this to be the one that, vis-à-vis other possibilities, is imposed on political and administrative decisionmaking processes. These objectives could not be achieved without examining the social structure in Extremadura and establishing the bases on which the present system of domination rests.

Keywords: Regional Inequality, Regional Policy, Development Models, Extremadura, Political Dependence. 



\section{INTRODUCCIÓN ${ }^{1}$}

Nuestro marco conceptual de referencia contempla los modelos de desarrollo y el análisis de sus peculiaridades desde diferentes disciplinas: la geografía, la sociología, la economía y el análisis territorial. Sin embargo, para nosotros, adquiere relevancia la Teoría de los Polos de Desarrollo y sus variantes tales como la Teoría del crecimiento equilibrado y la Teoría de la difusión espacial de las innovaciones (Posada, 1978), que son referencias necesarias para desentrañar las claves del «paradigma economicista» que se alza como el gran orientador de las políticas regionales. Forma parte también del marco conceptual de este trabajo el estudio de los principales elementos de la planificación regional en Extremadura ${ }^{2}$, que van desde el Plan de Desarrollo Regional (PDR 1989-93) y sus especificaciones para la región, plasmadas en el Plan de Desarrollo Regional para Extremadura (PDR-Extremadura de 1992), y los sucesivos Marcos de Apoyos Comunitarios (MAC). Estos planes dotan a la región de líneas de actuación política y de instrumentos de desarrollo regional que inciden en Extremadura desde la Unión Europea (Fondos Estructurales, Iniciativas Comunitarias, particularmente el LEADER y el INTERREG, la incidencia de la PAC...) ${ }^{3}$. Pero no sólo es esto; estas actuaciones se solapan con los instrumentos estatales de promoción regional (tales como los Incentivos Regionales, SODIEX y el Fondo de Cooperación Interterritorial - $\mathrm{FCl}-$ ), que en 1986 están en plena «reconversión», y los instrumentos regionales (SOFIEX, Corporación Industrial, PRODER), que en ese momento comienzan a readaptarse a las exigencias de una España integrada en la Unión Europea en la que las regiones cobran cada vez un mayor protagonismo. Por consiguiente, el modelo de desarrollo regional será subsidiario de los procesos en que se enmarcan esos instrumentos de incentivos regionales y que son el sustento del paradigma economicista que condiciona las posibilidades de maniobrabilidad de los responsables políticos y técnicos en el nuevo periodo autonómico (Escudero Araujo, 1995).

\section{HIPÓTESIS Y PROCEDIMIENTO DE INVESTIGACIÓN}

El marco teórico que hemos analizado, del cual en el epígrafe anterior se da una somera idea, tuvo la virtualidad de permitirnos obtener algunas deducciones hipotéticas en rela-

\footnotetext{
1 Este trabajo resume el procedimiento y conclusiones de la investigación social realizada a partir del análisis de datos secundarios y entrevistas cualitativas que sirvieron de base para la defensa de mi Tesis Doctoral en Universidad de Extremadura. Debo agradecer la eficaz y atenta dirección del Profesor Dr. D. Julián Mora Aliseda y, en todo lo concerniente al desarrollo regional de Extremadura, la indispensable aportación sociológica del Profesor Dr. D. José Antonio Pérez Rubio.

2 Materiales que resultan altamente clarificadores en este aspecto son los propios Marcos Comunitarios de Apoyo, el Libro Blanco sobre crecimiento, empleo y competitividad (1993), los Programas operativos del INTERREG (1994-99) y el documento de la Dirección General de Planificación La planificación regional y sus instrumentos (1992).

3 Ver De las Heras Borrero (1993) y Beltrán Fernández (1994), entre otros.
} 
ción al desarrollo regional y sus efectos en la estructura social de Extremadura, y que hemos estructurado del siguiente modo:

Primera: sobre el desarrollo regional aplicado en regiones atrasadas como Extremadura desde 1986 ejercen una influencia determinante diferentes aspectos de lo que hemos dado en llamar el «paradigma economicista del crecimiento», basado en una orientación hacia el exterior del sistema productivo, la desregulación de las relaciones sociales y la supeditación de la economía regional a los dictados de los países y regiones centrales.

Segunda: frente a esto (más bien al lado) se producen experiencias de desarrollo local endógeno que inicialmente son escasas y mal dotadas, además de excesivamente experimentales, inconexas y distribuidas anárquicamente por el territorio a partir del principio implícito de actuar sobre las comarcas que ya contaban con ciertas ventajas como, por ejemplo, disponer de una trayectoria de cooperación entre agentes sociales o de una infraestructura agroindustrial más desarrollada, etc. En estos casos el «efecto demostración» debía ejercer una mayor capacidad de propagación, aunque posteriormente se haya tendido a generalizar estas iniciativas aplicándolas miméticamente en todo el territorio.

Tercera: pese a los flujos económicos y a los recursos puestos a disposición, persisten desigualdades económicas, sociales y territoriales dentro de la región y en relación con nuestro contexto nacional y europeo, que constituyen el principal exponente de las limitaciones de estos procesos de desarrollo.

Cuarta: las estrategias de desarrollo, hasta finales de los años noventa, se han orientado hacia las dotaciones en infraestructura y, en cambio, se ha prestado una atención marginal a la revalorización de los recursos humanos.

Quinta: el impacto de estas políticas ha sido muy limitado en términos relativos. En su aspecto cualitativo, estos procesos han conducido a la región a una posición que hemos denominado como de «dependencia subsidiada».

Sexta: la estructura social, económica y espacial de la región se mantiene en una posición de transición con predomino de lo tradicional, cuyo paso definitivo a la etapa de «modernización» queda pendiente de una reestructuración social que debe hacerse a partir de la potenciación de los recursos humanos.

Este conjunto de hipótesis se ha utilizado para focalizar la investigación en una serie de aspectos muy concretos referidos al problema del desarrollo; las herramientas utilizadas para 
tratar de verificar las hipótesis fueron, de un lado, el tratamiento de la bibliografía y de los datos relevantes sobre el desarrollo regional, que se ha complementado con la indagación cualitativa, utilizando para ello una serie de entrevistas en profundidad a diferentes actores relevantes del proceso de implementación de las políticas de desarrollo en Extremadura, lo que nos permitió constatar la prevalencia de un modelo predominante frente a otros posibles. Para la interpretación de los datos nos fueron de utilidad las enseñanzas de la sociología general compendiada por Rocher (1980), pero sobre todo los análisis sociológicos del desarrollo de Extremadura del Profesor Pérez Rubio (1991, 1993, 1997). Este referente inicial hace posible que afrontemos el análisis de los datos cuantitativos con una conciencia analítica crítica.

De otro lado, hemos trabajado con bases de datos e instrumentos ya diseñados por el Departamento de Geografía y Ordenación del Territorio de la Universidad de Extremadura. Así, por ejemplo, el análisis de los impactos sobre el mercado regional de las políticas de incentivo, la evolución de ocupaciones en la estructura productiva regional y local, y los efectos sociales de algunos indicadores, económicos y territoriales, fueron estudiados a la luz de los trabajos de Mora Aliseda (1992), quien utiliza el análisis factorial de componentes principales aplicado sobre una Matriz de Información Geográfica (MIG) que contiene las unidades de análisis de los 380 términos municipales de Extremadura. Para realizar el tratamiento descriptivo de los datos de la matriz, el instrumento concluye agrupando las variables o atributos de los términos municipales en cinco bloques: en el primero de ellos se incluyen las variables físicas del territorio; en el segundo, las variables económicas; en el tercero, las variables de carácter social, que incluyen también las relativas a la formación del capital humano, entre otras; por último, las agrupaciones cuarta y quinta incluyen a las variables del mercado laboral y las de tipo cultural. El análisis factorial permite obtener una clasificación del territorio en base al peso que tiene cada uno de los factores.

Para el estudio de los desequilibrios territoriales internos de la región hemos recurrido a los trabajos de Sánchez Zabala (1992), que complementa el análisis factorial con la teoría de grafos. Esta herramienta permite conocer las peculiaridades de las redes de transporte, las conexiones y densidades de los núcleos urbanos en función de ciertos parámetros; aporta, además, un conjunto de índices con los que poder analizar la estructura de redes. Los trabajos de estos autores tienen la virtualidad de conjuntar el estudio espacial y el análisis de los efectos sociales que éste puede tener, lo que desde el punto de vista de nuestra indagación resultó sumamente pertinente, ya que pondera adecuadamente los factores sociológicos y los geográficos. 


\section{MODELO DE INTERRELACIONES}

Creemos que nuestro principal hallazgo es ofrecer una explicación de la tramoya del desarrollo regional de Extremadura a finales del siglo pasado y que, en síntesis, se puede definir como la de un sistema de interrelaciones cuyos elementos se encuentran en situación de desequilibrio debido a diversos factores de orden histórico y social que las inversiones de finales de siglo no han conseguido equilibrar.

La figura 1 (Sistema de interrelaciones del modelo extremeño de desarrollo) permite obtener un visión gráfica del modelo de desarrollo. Como puede apreciarse en ella, los flujos de diverso signo y orientaciones que emanan de las políticas de desarrollo aplicadas en la región inciden de forma diferente en cada parte del sistema regional. Así, por ejemplo, las inversiones para la vertebración territorial se destinan, esencialmente, a las dotaciones de la red viaria y al equipamiento social ${ }^{4}$; pero, en cambio, no se aborda el problema de la ordenación del territorio en un contexto regional en el que la pérdida de peso y la desestructuración de las comarcas tradicionales y de sus cabeceras son una fuente importante de los desajustes que se explican en la tabla 1 (Síntesis de los condicionantes y sus efectos para el periodo 1986-1999), que provocan, por ejemplo, la pérdida de eficacia de los proyectos de desarrollo 5 .

En general, la insistencia en las inversiones en este tipo de infraestructuras como estrategia política dominante ha tenido como resultado un desarrollo espectacular de tres ciudades principalmente: Badajoz y Cáceres — las capitales de provincia-y Mérida —capital de la Comunidad Autónoma- (Campesino, 1995). Los efectos polarizantes (en términos de creación de riqueza, expansión demográfica, inversiones de capital privado, etc.) de esta estrategia de desarrollo se han sobrepuesto a los efectos difusores que pretendidamente debería tener. En consecuencia, han aumentado los desequilibrios territoriales en el interior de la región.

Los inputs que el sistema incorpora destinados a la modificación de los factores normativos y la formación del factor humano han tenido, en el periodo analizado, un alcance bastante limitado, que calificamos como «flujos de media intensidad». Es cierto que en los planes de desarrollo se contemplan partidas para estos campos de acción, pero, dado el cúmulo de necesidades de la región en este campo, esto resulta insuficiente. Las partidas incluidas en

\footnotetext{
${ }^{4}$ Ejemplo de estas inversiones en equipamiento social son los polideportivos, piscinas públicas, casas de culturas, etc., que se han ido construyendo en la mayor parte de los municipios de la región.

5 A este respecto pueden consultarse Vázquez Barquero (1998), Soto (1995), Salvá Tomás (1997) y García Dory (1992), entre otros autores.
} 


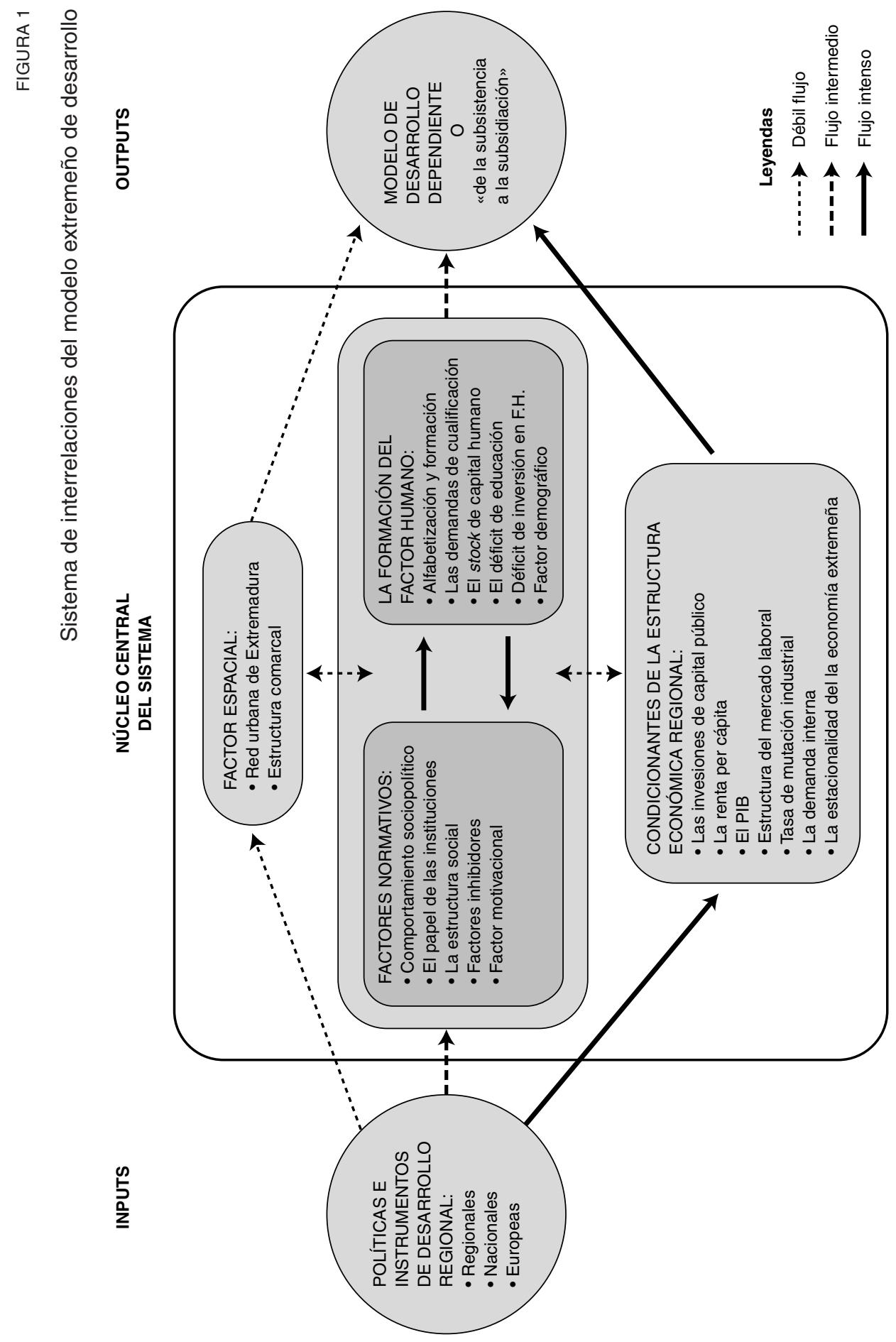


este concepto se destinan prioritariamente al complemento de rentas, lo que es un aspecto muy parcial del «desarrollo del factor humano" y, por consiguiente, no ha logrado corregir las disparidades que existen en este complejo subsistema en comparación con los parámetros estatales y europeos.

El resultado es un tipo de desarrollo que privilegia los aspectos economicistas (Pérez Rubio, 1991) y minusvalora otros subsistemas sobre los que se llega a incidir de manera sesgada. Como consecuencia de lo anterior, el modelo de desarrollo es demasiado dependiente de estos flujos, de manera que de eliminarse éstos la capacidad de mantener este modelo sería remota.

Las actuaciones de desarrollo durante este periodo adolecieron de una estructura organizativa ad hoc, lo que en parte impide obtener respuestas suficientemente solventes en la perspectiva de lograr un mejor aprovechamiento de sus potencialidades y dar así posibilidades de combatir el modelo subsidiado de desarrollo. Este último aspecto se debe, en buena medida, a que no existe una estructuración de las interdependencias internas del sistema (relaciones recíprocas entre las diferentes partes implicadas), lo que origina una evidente pérdida de la sinergia que, generalmente, se asocia a todo proceso integrado de desarrollo.

A continuación se detallan los condicionantes que afectan a cada subsistema de la estructura regional. En la tabla 1 se pueden apreciar aquellos aspectos que los procesos de desarrollo deberían haber mejorado en la región y que, sin embargo, a finales de los noventa aún no se habían visto alterados sustancialmente. 


\section{Síntesis de los condicionantes y sus efectos para el periodo 1986-1999}

Subsistema

Factores condicionantes que no han sido sustancialmente modificados por las acciones de desarrollo regional
Subsistema territorial ${ }^{6}$ de Extremadura

- La red urbana es comparativamente mínima y embrionaria. Jerárquicamente desequilibrada e incapaz de generar intercambios fluidos entre los nodos más relevantes.

- La posición de la región es periférica y fronteriza. Las regiones de frontera constituyen zonas económica y socialmente deprimidas. Esta posición de desventaja comparativa afecta particularmente a las comarcas del noroeste de la región, en situación de progresivo declive.

- La estructura comarcal de la región se ha caracterizado en los últimos decenios por la ruptura de las bases de la comarca tradicional.

Subsistema normativo de Extremadura

- El familismo amoral ${ }^{7}$ siguió teniendo vigencia en la región con la constitución de la nueva "clase burocrática» que surge con la formación de las instituciones autonómicas. Esto se solapa con comportamientos clientelares atávicos presentes aún en importantes zonas rurales.

- El proceso de socialización que se trata de imponer, basado en la economía capitalista occidental y tecnocrática, encuentra fuertes resistencias entre la población: frente a los criterios universalistas y racionalistas de organización de la sociedad y de sus instituciones, persisten los criterios particularistas y la mentalidad tradicional ${ }^{8}$.

- Persisten aspectos motivacionales anacrónicos tanto de la clase trabajadora como entre la clase empresarial ${ }^{9}$ y las organizaciones intermedias. La capacidad de asumir riesgos o el afán de logro son factores motivacionales poco arraigados.

- La estructura social se encuentra en un proceso de transición inacabado hacia una sociedad «moderna», donde la clase trabajadora se localiza en el sector servicios, caracterizado por la «terciarización prematura»; las clases medias está muy vinculadas a los servicios públicos, y las clases altas son escasas y asociadas a actividades profesionales y menos a las empresariales. Las organizaciones de clase tienen aún escasa implantación social ${ }^{10}$.

Subsistema de la formación del factor humano ${ }^{11}$
- La desviación de la productividad regional (productividad de los empleados) respecto de la media nacional se cifra en $-26,24 \%$. La parte de este porcentaje que es atribuible al déficit de formación y educación es el 8,33\%, de tal forma que de compensarse este aspecto la desviación de la productividad regional respecto de la estatal se reduciría al $17,91 \%$.

- Inapreciable demanda de cualificación del factor humano en comparación con otras regiones del Estado, tan sólo asimilable a lo que ocurre en Galicia, y que está asociado a una escasa tasa de mutación industrial.

6 Ver Sánchez Zabala (1992), Mora Aliseda (1991), Parellada i Sabata y García Brosa (1998), entre otros.

7 Ver Porras Nadales (1993).

8 Ver Alonso (1992).

9 Ver Palazuelo Manso y Pérez Rubio (1996).

10 Ver Pérez Rubio (1991, 1997).

11 Ver Ramajo Tejeda (1997: 47), Mitch (1993), Remedios Solís (1997) y Beneyto y Guillén (1998), entre otros. 
TABLA 1

\section{Continuación}

Subsistema
Factores condicionantes que no han sido sustancialmente modificados por las acciones de desarrollo regional
Subsistema de la formación del factor humano

- Se constatan índices negativos del stock de capital humano y elevado desaprovechamiento del capital humano disponible; en este sentido, se observan desajustes entre las demandas y las ofertas de titulados en el mercado laboral regional.

- Las tasas de titulados a todos los niveles son inferiores a la media estatal, y en el ranking de regiones se sitúa en las posiciones más bajas.

- Los niveles de alfabetización son muy inferiores en todos los niveles al resto del Estado.

- Del análisis de las Acciones de los Marcos de Apoyo Comunitarios (MAC) se desprende que el 17\% del MAC 94-99 se destina a este capítulo, pero el subeje que aglutina la mayor parte de los recursos es el de atención a desempleados (76\%) y tan sólo el 16,4 de los recursos se destinaron a la formación de trabajadores.

Subsistema económico ${ }^{12}$ regional
- Inversión del capital público: las regiones como la extremeña presentan menores niveles efectivos de equipamiento público y, consecuentemente, son las más pobres, y el rendimiento esperado de la inversión es generalmente bajo.

- Renta regional: el bajo nivel de desarrollo que correspondería a Extremadura por su producción se ve corregido en buena medida por el efecto de las transferencias recibidas de fuera de nuestra región.

- Producto interior bruto (PIB): desde la integración en Europa, el PIB regional es de los que experimentan un mayor crecimiento en términos absolutos. Pese al incremento de nuestro PIB, la posición que Extremadura ha ocupado en este ranking ha sido la última.

- Factor demográfico ${ }^{13}$ : estancamiento demográfico y recesión en las comarcas del noroeste. Saldo migratorio negativo en las dos últimas décadas. Elevadas tasas de envejecimiento y de los índices de dependencia.

- Mercado de trabajo con graves deficiencias estructurales: tasas de desempleo elevadas que no se han corregido en las últimas dos décadas y que se mantienen constantes en torno al $17 \%$ de la población activa. Nivel de activos bajo y alto el de las tasas de inactivos.

- Demanda interna raquítica asociada a una renta disponible de las más bajas del Estado. Es una demanda polarizada en bienes de subsistencia.

- Inversiones en I+D: suponen el 0,39\% del PIB regional, frente al 0,94\% de la media española, o el $2,8 \%$ de Alemania.

- La supeditación a la estacionalidad: la evolución del índice de vivacidad elaborado por el Anuario del Mercado Español (AME), por ejemplo, pone de manifiesto el sometimiento a los factores coyunturales que padece la región.

12 Ver Ramajo Tejeda (1997) y García Pérez (1996), entre otros.

13 Ver Gurria Gascón (1997). 


\section{MENCIÓN EXPRESA A LOS RECURSOS HUMANOS}

El tratamiento que las políticas de desarrollo han hecho del factor humano es objeto central de nuestra investigación, que, en su pretensión última, trata de demostrar que la supeditación de la región a las regiones centrales se basa en la falta de eficacia de las políticas llevadas a cabo en este campo.

El sistema productivo regional genera una demanda formativa del factor humano muy débil e inespecífica cuyo principal efecto es que el paso de «recursos humanos disponibles» a "stock de capital humano", propio de un sistema productivo dinámico, sea retardado o no se llegue a producir en algunos sectores y ramas ${ }^{14}$.

En regiones como la extremeña existe más población que la media con niveles inferiores de instrucción y menos que la media con niveles superiores de instrucción. Los indicadores que hemos manejado revelan una inadecuación general del sistema formativo regional en su relación con la inserción laboral (Ortiz Chaparro, 1997).

Los déficits respecto de la revalorización de los recursos humanos se deben vincular no sólo a una estructura productiva, sino también al papel secundario que han tenido las inversiones en capital humano. Por ejemplo, como se ha señalado en otro momento, el Marco de Apoyo Comunitario de 1994 a 1999 (MAC 1994) destinó el 17\% de sus recursos a "valorización de los recursos humanos», un porcentaje que puede, en sí mismo, considerarse bajo para las necesidades detectadas en este campo en Extremadura. Sin embargo, lo que queremos destacar es la composición de esta partida, que se muestra en el gráfico 1.

Como se puede apreciar, el capítulo dedicado a la «inserción y reinserción de desempleados", con un $76 \%$ de las inversiones, acumula la mayor parte de estas partidas, frente al 24\% que se destinó al resto de conceptos: «la formación continua», «la formación ocupacional» y el «equipamiento físico para la formación». Por ejemplo, la formación profesional, a pesar de ser un factor clave para el desarrollo regional al flexibilizar el mercado de trabajo y adecuar la oferta a la demanda, no contó con la implantación necesaria en la región en el periodo analizado.

Conviene recordar aquí, respecto de los condicionantes de la inversión en recursos humanos dentro de una política regional centrada en el desarrollo, que el crecimiento económico de un territorio parece depender del incremento del capital por trabajador, considerando el capital en cuatro formas: capital en equipamientos privados, capital en infraestructuras, ca-

14 Ver INE (1997: 367, 422, 450), Sandberg (1993), Ramajo Tejeda (1997) y Dabán Sánchez y Murgi García (1997). 
GRÁFICO 1

Distribuciones de inversión en recursos humanos en el MAC 1999 (en porcentajes)

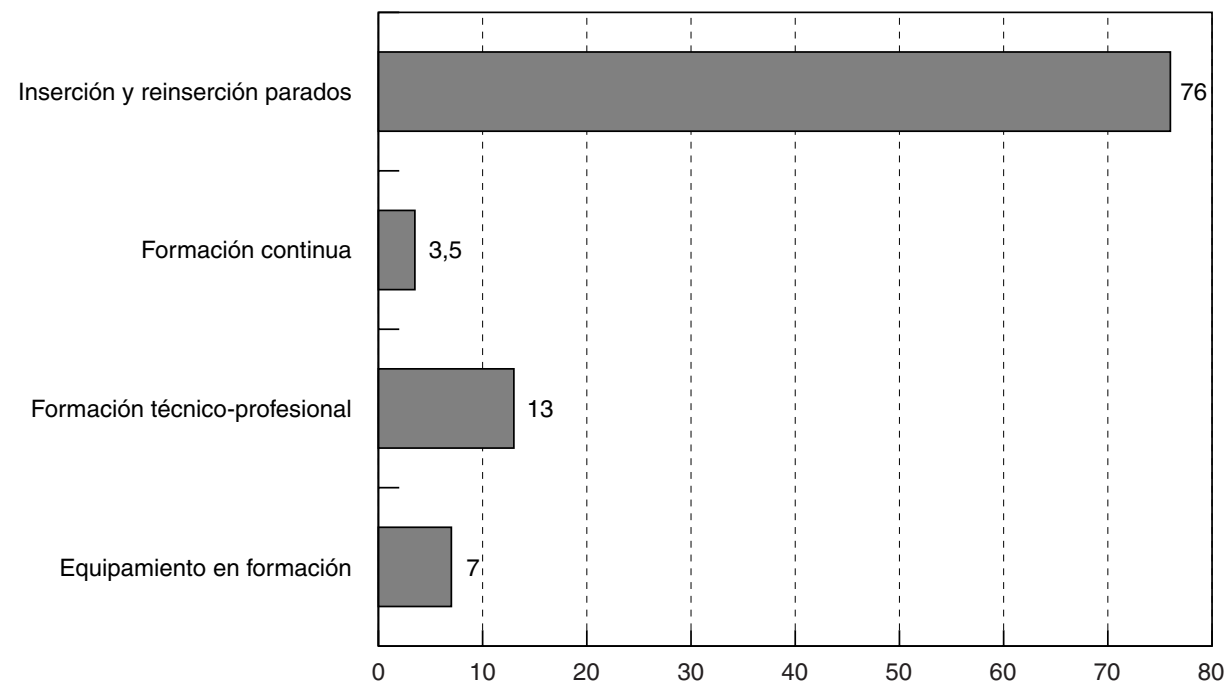

FUENTE:

Elaboración propia a partir de datos del MAC 1999.

pital humano y capital tecnológico (Investigación y Desarrollo - I+D-e innovación). Estas cuatro formas han de ir aparejadas, porque la interacción de unas con otras parece poseer conjuntamente efectos externos, si no rendimientos crecientes. Inversiones de un tipo no acompañadas de las restantes pueden limitar la eficaz asignación de los recursos (Myro Sánchez, 1996).

El sesgo economicista de las políticas de desarrollo tiene un último reflejo en el peso que adquieren los diversos subfactores que componen las inversiones públicas en la región. El gráfico 2 ofrece la trayectoria seguida por las inversiones públicas destinadas a Extremadura a lo largo de los años que van desde 1980 a 1993. Para este tratamiento se han agrupado los datos en dos grandes categorías: las «inversiones en infraestructuras económicas", esto es, todas aquellas referidas al sistema productivo y a las infraestructuras físicas, y, por otro lado, las «inversiones destinadas a aspectos sociales», tales como la educación, formación del capital humano, los servicios sociales, etc. Como puede apreciarse, las inversiones públicas en «infraestructura social» han venido perdiendo peso en relación al 
GRÁFICO 2

Inversiones en infraestructura económica y social en Extremadura (1980-1993) (en porcentajes)

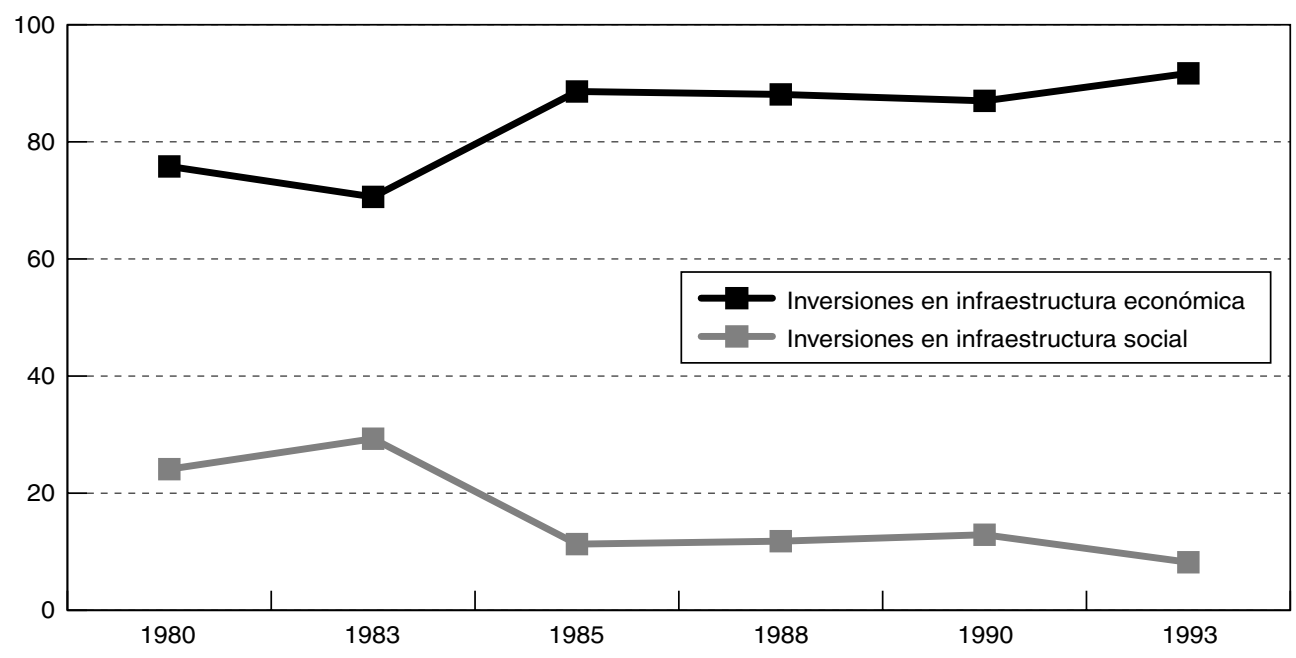

FUENTE:

Elaboración propia a partir de Delgado Rodríguez (1998).

otro grupo en las últimas décadas del siglo pasado, llegando a representar a mediados de los años noventa en torno al $10 \%$ del conjunto de las inversiones públicas que se realizaban en la región.

\section{CONCLUSIONES}

A lo largo del trabajo se llega a conclusiones que en algunos casos resultan desalentadoras para los esfuerzos de desarrollo de las regiones periféricas; si bien advertimos que en el último lustro se observan correcciones hacia el incremento de inversiones en la formación del factor humano, con importantes innovaciones de tipo cualitativo en este ámbito.

Del periodo analizado se concluye que se ha partido de una multiplicidad de modelos de desarrollo, con una evolución cronológica e ideológica determinada, aunque con la prevalencia del paradigma economicista, dentro del cual y a modo de ornamento se han desa- 
rrollado otras experiencias complementarias, a veces contradictorias, encuadradas en la corriente del desarrollo endógeno. Esa situación de partida cristaliza en el modelo actual, que no está logrando eliminar las desigualdades regionales e intrarregionales, que era la pretensión explícita de los Fondos Estructurales de la Unión Europea (Iniesta Alonso-Sañudo, 1993). De modo que las desigualdades entre las regiones «ricas» y «pobres» se mantienen en términos relativos, sometidas las últimas a dinámicas de subsidiación y a la dependencia (Sánchez-Oro Sánchez, 1995).

Es conocido que la política regional europea para las áreas atrasadas se instrumentaliza a través de los Fondos Estructurales. Estas actuaciones, en regiones como Extremadura, se suman, se superponen o se complementan con actuaciones regionales de la Administración estatal —sometida durante las dos últimas décadas del siglo xx a una irremediable pérdida de relieve- y la autonómica ${ }^{15}$. Esta adición de iniciativas generó un complejo, disperso y descoordinado conjunto de instrumentos de desarrollo regional. El impacto de estas políticas, medido a través de los indicadores al uso ${ }^{16}$, ha resultado ser escaso y desigual. Esto se advierte en el desarrollo desigual de los subsistemas de la región considerados de forma separada: en términos relativos, la estructura social y económica de Extremadura ha experimentado pocas variaciones; en términos absolutos se observan avances notorios, fundamentalmente en el campo de las infraestructuras y dotación de equipamientos.

Las salidas de la situación de estancamiento y dependencia pasa por lo que hemos denominado una reestructuración social desde la potenciación de los recursos humanos. Para ello hay que completar el proceso de transición hacia la modernidad y superar lo que denominamos las bases internas y externas de la dependencia.

Desde este análisis constatamos que son precisamente los recursos humanos la base del desarrollo regional, aunque detectamos un importante déficit en las cualificaciones, en los niveles de instrucción y una importante quiebra en la articulación de los sistemas formativos con el mercado laboral, que hace problemática la conversión de los recursos humanos en capital humano (Sandberg, 1993). Las propuestas para un desarrollo sostenible deben considerar otros elementos para que medidas de carácter económico y medioambiental sean eficaces. Los factores socioculturales, demográficos y territoriales inciden directamente en el desarrollo regional. Es necesario reorientar la actual estructura territorial me-

\footnotetext{
15 Un intento de dotar de una estructura administrativa autonómica armonizadora del conjunto de acciones de desarrollo lo constituye la creación en Extremadura por primera vez en 2003 de la Consejería de Desarrollo Rural. Desde el punto de vista de la institucionalización académica del estudio del desarrollo regional es de destacar, por su carácter de pionero, al Centro de Desarrollo Sostenible, promovido por la Facultad de Empresariales y Turismo de la Universidad de Extremadura.
}

${ }^{16}$ CESEx'94, BBV (1997), Comisión Europea (1994) y Anuario Estadístico de la Junta de Extremadura, entre otros. 
diante reformas que permitan un aprovechamiento sostenible de casi el único elemento importante y ventajoso que tiene Extremadura, como es el territorio. Ello pasa por un plan estratégico de desarrollo a escala comarcal.

Queremos terminar con la reflexión de Samuelson y Nordhaus (1986: 1003) sobre el problema del desarrollo, y que no cierra este debate, sino, al contrario, nos sugiere la necesidad de encontrar en nuestro propio tejido social la forma de escapar de la dependencia: «Cuando se leen historias como la de Japón o Estados Unidos, tal vez parezca que la adaptación de la tecnología extranjera es una fácil receta para el desarrollo. Quizá el lector se diga: vete al extranjero y copia los métodos más eficientes; ponlos en marcha en tu país y luego siéntate a esperar a que salga la producción adicional. Naturalmente, esto no funciona precisamente así. Unos pocos expertos pertrechados de una lista de proyectos no pueden resolver todos los problemas de un país (o región) pobre. Hay miles de barreras culturales y económicas que se oponen al progreso. La experiencia demuestra que para que las tecnologías funcionen, se necesitan personas que recojan esas ideas y las empleen».

\section{BIBLIOGRAFÍA}

ALONSO, Luis Enrique (1992): «Un viaje hacia la cara oscura de la Modernización», Revista Agricultura y Sociedad, n. ${ }^{\circ}$ 62, Ministerio de Agricultura, Pesca y Alimentación, Madrid.

BBV (1997): La renta de España y su distribución provincial.

BELTRÁN FERNÁNDEZ, C. (1994): «El desarrollo rural y la iniciativa comunitaria "LEADER” en España», Revista Papeles de Economía Española, n. ${ }^{\circ s} 60-61$, Fundación FIES.

BENEYTO y GUILLÉN (1998): Formación profesional y empleo: la construcción de un nuevo modelo, Ed. Germanía.

CAMPESINO, A. (1995): «El sistema de ciudades en Extremadura», Situación, n. ${ }^{\circ} 3$.

CESEx'94: Informe sobre la situación socioeconómica de Extremadura, Consejo Económico y Social de Extremadura (multicopiado).

COMISIÓN EUROPEA (1994): Competitividad y cohesión: las tendencias de las regiones, V Informe Periódico.

DABÁN SÁNCHEZ y MURGUI GARCÍA (1997): “Convergencia y rendimientos a escala en las regiones españolas: la base de datos BD.MORES», Información Comercial Española (ICE), n. ${ }^{7}$ 762, Ministerio de Economía y Hacienda, Madrid.

DE LAS HERAS BORRERO, F. M. (1993): «Los fondos estructurales de la Comunidad Europea y su incidencia en el desarrollo de Extremadura», en Desarrollo Regional de Extremadura, Cámara de Comercio de Cáceres.

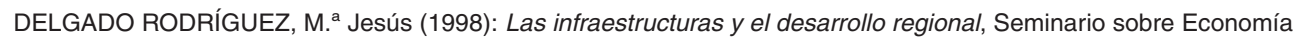
Extremeña, 30 y 31 de enero, Universidad de Extremadura, Papeles de Economía Española, Fundación FIES.

DIRECCIÓN GENERAL DE PLANIFICACIÓN (1992): La planificación regional y sus instrumentos, Ministerio de Economía y Hacienda. 
ESCUDERO ARAUJO, L. (1995): «La política regional comunitaria y Extremadura: balance de una nueva etapa (1989-1999)», en La agricultura y la ganadería extremeñas en 1994, Caja de Badajoz.

FRADES GASPAR, D., y otros (1995): «El programa LEADER I en Extremadura», en La agricultura y la ganadería extremeñas en 1994, Caja de Badajoz.

GARCÍA DORY, M. A. (1992): «El programa LEADER en España», Documentación Social, n. ${ }^{\circ}$ 87, Cáritas Española, Madrid.

GARCÍA PÉREZ, Juan (1996): «Desde la historia a la prospectiva. Un análisis sobre el pasado reciente y el futuro inmediato de la economía extremeña», en Programar la esperanza, Editora Regional de Extremadura.

GURRIA GASCÓN, J. L. (1996): Población y dinámica demográfica en Extremadura 1990-2015. Curso sobre demografía del Plan de Formación de la Junta de Extremadura (material multicopiado).

INE (1997): Indicadores sociales de España.

INIESTA ALONSO-SAÑUDO, A. (1993): «Política de cohesión y fondos estructurales», Revista Documentación Social, n. ${ }^{\circ} 91$, p. 215, Cáritas Española, Madrid.

Libro Blanco sobre crecimiento, competitividad, empleo. Retos y pistas para entrar en el siglo xxı, Comisión de las Comunidades Europeas. Bruselas, 5 de diciembre de 1993.

Marco Comunitario de Apoyo para el desarrollo y el ajuste estructural de las regiones menos desarrolladas. Objetivo n. ${ }^{\circ}$ 1. 1994-1999, Comisión de las Comunidades Europeas, Dirección General de Políticas Regionales.

MYRO SÁNCHEZ, R. (1996): «Estrategias y políticas para el desarrollo económico regional», en La industria de una región no industrializada: Extremadura, 1750-1990, UEX.

MITCH, D. (1993): «Educación y crecimiento económico: ¿Otro axioma de indispensabilidad? Del capital humano a las capacidades humanas», en La maldición divina. Ignorancia y atraso económico en la perspectiva histórica, Alianza Universidad.

MORA ALISEDA, J. (1991): «El modelo de asentamientos humanos en Extremadura», Revista Estudios Territoriales, n. ${ }^{\circ}$ 36, pp. 129-145, Universidad de Málaga.

- (1992): La organización del territorio extremeño: economía, población y medio físico, FUNDICOT-Extremadura y Universidad de Extremadura.

ORTIZ CHAPARRO, Francisco (1997): «El crecimiento sin empleo: trabajo y empleo en la nueva sociedad», en «Informática, Información y Comunicación», Documentación Social, n. ํ107, Cáritas Española, Madrid.

PALAZUELO MANSO y PÉREZ RUBIO (1996): «Mentalidad empresarial y perspectivas económicas en Extremadura a finales de la década de los 80", en La industria de una región no industrializada: Extremadura, 1750-1990, UEX.

PARELLADA I SABATA y GARCÍA BROSA (1998): «Las disparidades regionales españolas en el seno de la Unión Europea», en Economía y política regional en España ante la Europa del siglo XXI, Akal.

PÉREZ RUBIO, José Antonio (1991): “Consideraciones sobre las perspectivas de análisis del subdesarrollo y la importancia que otorgan a las relaciones sociales", Desarrollo Regional. Revista Alcántara, n. ${ }^{\circ}$ 22, Excma. Diputación Provincial de Cáceres.

- (1993): «Formas de aproximación a la estructura social de Extremadura», en Desarrollo Regional de Extremadura, Cámara de Comercio de Cáceres.

- (1997): Actores en el desarrollo de las regiones periféricas. Un planteamiento de la acción de las clases y el Estado en las regiones periféricas de España y Portugal (material multicopiado). 
PÉREZ RUBIO, J. A., y SÁNCHEZ-ORO SÁNCHEZ, M. (1998): Notas sobre el desarrollo rural en España. Perspectivas desde la sociología, Resúmenes de comunicaciones y ponencias, VI Congreso Español de Sociología.

PORRAS NADALES (1993): «Representación Política y Clientelismo: El caso de Andalucía», Revista Fomento Social, $\mathrm{n}^{\circ} 47$, p. 495.

POSADA, Luis Javier (1978): «Fundamentos económicos-espaciales de la teoría de Centros de Desarrollo», Agricultura y Sociedad, n. ${ }^{\circ}$, Ministerio de Agricultura y Comercio.

Programa de Desarrollo Regional de Extremadura (1992-1995), tomos I y II (material multicopiado).

Programa Operativo ADAPT (1994) (material multicopiado).

RAMAJO TEJEDA (1997): Debilidad industrial y atraso económico en Extremadura, Asamblea de Extremadura.

REMEDIOS SOLÍS, E. (1997): Formación Profesional, situación actual y entorno productivo, Cámara de Comercio e Industria de Cáceres.

ROCHER, G. (1980): Introducción a la sociología general, Herder, Barcelona.

SALVÁ TOMÁS, Pere (1997): Nuevos desequilibrios regionales: un debate en vísperas del 2000. Cambios Regionales a finales del siglo $x x, \mathrm{AGE}$, Universidad de Salamanca.

SÁNCHEZ-ORO SÁNCHEZ, M. (1995): El desarrollo regional extremeño y sus instrumentos en el marco de la Unión Europea. Trabajo investigación 3. ${ }^{\text {er }}$ ciclo. Material inédito.

SÁNCHEZ ZABALA, R. (1992): Comarcalización funcional y ordenación del territorio en Extremadura, Universidad de Extremadura.

SANDBERG, Lars G. (1993): «Ignorancia, pobreza y atraso económico en las primeras etapas de la industrialización europea: variaciones sobre el gran tema de Alexander Gerschenkron», en La maldición divina. Ignorancia y atraso económico en la perspectiva histórica, Alianza Universidad.

SAMUELSON y NORDHAUS (1986): Economía, McGraw-Hill.

SOTO, P., y GÓMEZ, L. (1995): «Algunos retos para el desarrollo rural en España. Reflexiones en torno a un caso: el Valle del Jerte», Agricultura y Sociedad, n. ${ }^{\circ} 77$, pp. 173-198, Ministerio de Agricultura y Comercio. 